run, to be a failure. But it will be a useful failure if it serves to show up false assumptions which we tacitly make. Some, such as the existence of female gametes, are relatively trivial. Others are much more deeply involved in our thought, and if they are false the results are much more serious.

Thus, Dr. Woodger assumes that a cell can be divided into momentary parts, that is to say that in theory, at least, we can specify the state of a cell at any instant with no limit to our accuracy. This is untrue for an atom, and, if it is correct that change is part of the very essence of life, it may turn out to be even more glaringly untrue of the cell.

Again, it is assumed that certain cell-parts, for example, genes, are individuals each with one and only one ancestor at any past date. This assump. tion has so far satisfied geneticists. But actually when two like genes come together in a homozygote at pachytene, and after a time which may be as long as forty-five years, finally separate, it is impossible to say which of the separating genes represents a given one of the synapsing genes. The uncertainty may be small, but is not necessarily negligible, and may prove very important.

Similarly, it is assumed that genes divide. This may be true. It is equally plausible that they reproduce by copying rather than by division.

The value of Dr. Woodger's book will be greatly enhanced if he clears it, so far as possible, both from definite myths, such as that of the female gamete, and from postulations of unobserved phenomena, such as gene division. If this is done, his method may prove valuable for morphologists for some generations to come, although until it takes cognizance of chemical facts it will be of little use to the biologist and none to the biochemist. But at present it falls between the two stools of positivistic description and materialistic explanation. I believe that the former ideal is in the long run unattainable. But the attempt to attain it has inspired such biologically valuable work as that of Karl Pearson.

In his preface Dr. Woodger emphasizes the tentative character of his book. If it proves possible to rewrite it in terms of observable phenomena only, without such postulates as gene divisions and momentary states of cells, it will be a valuable contribution to human thought. Until then, its main importance may be to lay bare erroneous assumptions rather than to serve as the basis of a further theoretical construction. J. B. S. HALDANE.

\title{
Synaptic Transmission
}

Autonomic Neuro-Effector Systems

By Prof. Walter B. Cannon and Prof. Arturo Rosenblueth. (Experimental Biology Series.) Pp. xiv +229 . (New York: The Macmillan Co., 1937.) 17s. net.

SEVERAL reviews have been written recently $S$ dealing with the chemical transmission of nervous impulses. A monograph has now been published which gives, for the first time, an authoritative exposition of the views of the Harvard School. The authors have both written many papers describing investigations of the properties of adrenergic nerves. They have shown that stimulation of these nerves causes the liberation of at least two different substances. The clear presentation of the evidence for this conclusion forms the most valuable and impressive feature of the book. No one could question the experimental results, but their interpretation is still in doubt. The theory that drugs combine with a special receptive substance in the motor organ provides the only reasonable explanation of the specificity of drug action, but Cannon and Rosenblueth's theory that this receptive substance is liberated with the drug into the blood stream seems unnecessarily complicated, and has met with opposition.

The book is argumentative. The authors reject Bacq's rather factious criticisms of their theory of sympathins $E$ and $I$. They reject the theory advanced by Monnier and Bacq to account for the failure of $933 \mathrm{~F}$ to antagonize the effect of adrenergic nerves in favour of that advanced by Dale and Gaddum to account for similar phenomena in connexion with atropine. They reject the theory which explains dose-effect curves in terms of individual variations of the sensitivity of muscle elements on the ground that it would be necessary to assume that the logarithm of the sensitivity of these elements was normally distributed. This is not the weak point of the theory in question, since the logarithms of biological quantities usually are normally distributed. They accept the theory which regards these curves as hyperbolæ, and interprets them in terms of mass action, but do not appear to realize that, on this theory, the ratio to one another of the potency of two solutions must be independent of the size of the doses used.

This book is, in fact, likely to lead to much discussion. 